\title{
MaxSynBio: Wege zur Synthese einer Zelle aus nicht lebenden Komponenten
}

Petra Schwille, * Joachim Spatz, Katharina Landfester, Eberhard Bodenschatz,

Stephan Herminghaus, Victor Sourjik, Tobias J. Erb, Philippe Bastiaens, Reinhard Lipowsky, Anthony Hyman, Peter Dabrock, Jean-Christophe Baret, Tanja Vidakovic-Koch, Peter Bieling, Rumiana Dimova, Hannes Mutschler, Tom Robinson, T.-Y. Dora Tang, Seraphine Wegner und Kai Sundmacher*
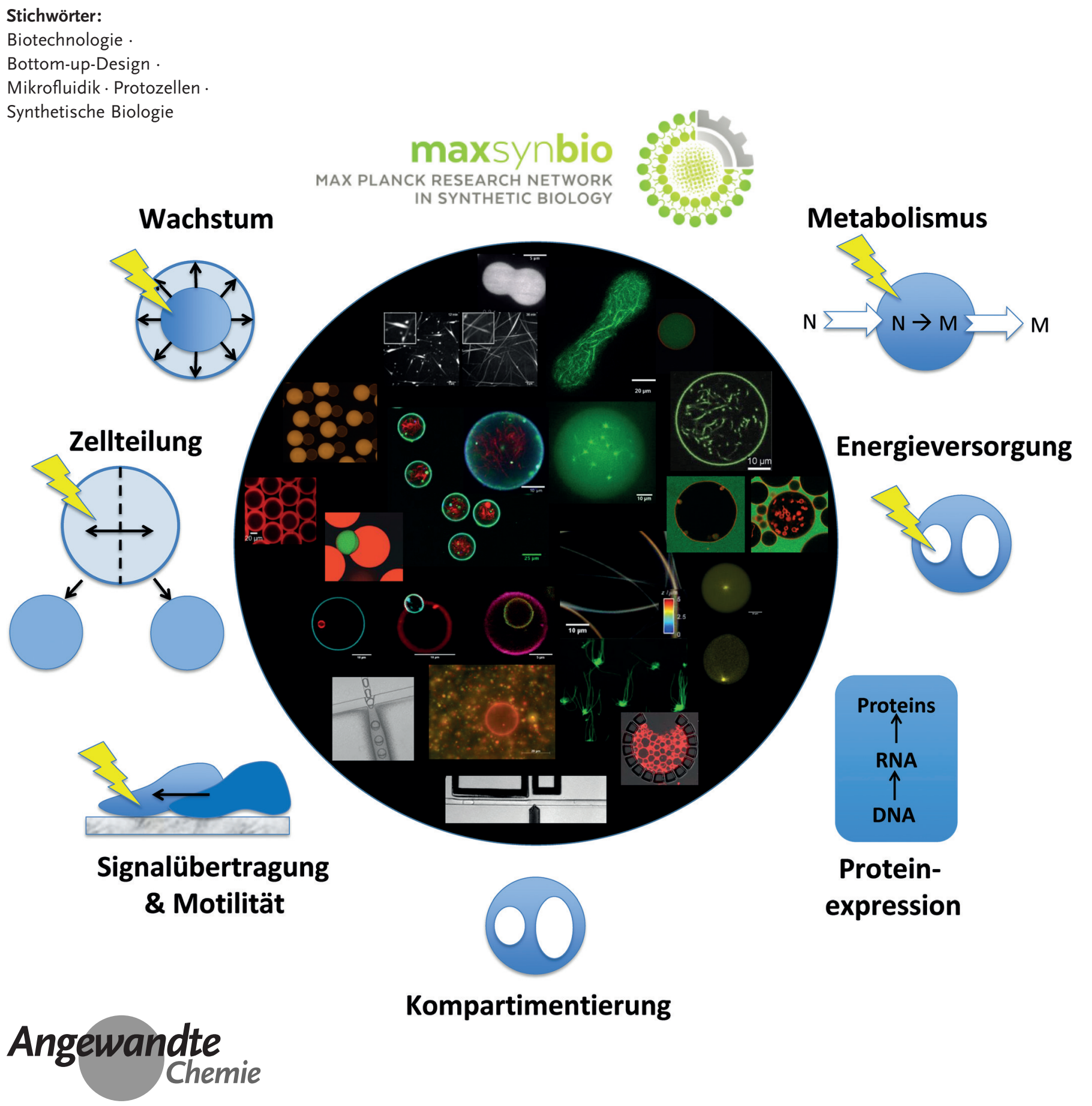
$\boldsymbol{E}_{\text {in }}$ großes deutsches Max-Planck-Forschungskonsortium („MaxSynBio") untersucht lebende Systeme aus fundamentaler Perspektive. Das Forschungsprogramm von MaxSynBio verfolgt dabei ausschließlich einen „Bottom-up“-Ansatz in der synthetischen Biologie: Es konzentriert sich auf die detaillierte Analyse und das Verständnis essentieller Lebensprozesse, indem es diese Prozesse als elementare Module in minimalen synthetischen Systemen rekonstituiert. Das ultimative Ziel von MaxSynBio ist es, eine lebende Zelle komplett aus nicht lebenden Komponenten zu konstruieren. Die grundlegenden Erkenntnisse aus den Aktivitäten in MaxSynBio können auf lange Sicht zur Etablierung neuer biotechnologischer Verfahren führen, die auf synthetischen Zellkonstrukten basieren. Langfristig könnten dadurch Organismen ersetzt werden, die derzeit in der konventionellen Biotechnologie Verwendung finden.

\section{Minimalzellen, künstliche Zellen und Protozellen in der synthetischen Biologie}

Der aufstrebende Forschungszweig der synthetischen Biologie gilt als eines der großen Versprechen für die Biotechnologie der Zukunft. Diese neuartige Herangehensweise an die Biologie ist teilweise inspiriert durch den großen Erfolg der synthetischen Chemie im vergangenen Jahrhundert, aber auch durch die Vielzahl mechanistischer Erkenntnisse, die über Jahrzehnte in der Molekularbiologie und Gentechnik gesammelt wurden. Derzeit ist die Biotechnologie begrenzt auf natürliche Produktionsorganismen, wie zum Beispiel mikrobielle Zellen, die enorm komplexe Systeme sind. Natürliche biologische Systeme bestehen aus einer Vielzahl funktioneller zellulärer Elemente und Netzwerken mit einer großen Anzahl von Komponenten, die eine inhärente Redundanz und funktionelle Mehrdeutigkeit aufweisen. Mithilfe synthetischer Biologie sollen einfachere biologische Gebilde generiert werden, die im Gegensatz zu natürlichen biologischen Systemen mit höchster Präzision vorhergesagt, manipuliert und gesteuert werden können.

Die Komplexität natürlicher biologischer Systeme kann als Produkt eines sehr langen evolutionären „Wettrüstens“ zwischen verschiedenen Lebensformen in der Konkurrenz um Ressourcen verstanden werden. Es ist jedoch keineswegs klar, ob das Leben als solches, einschließlich seiner grundlegenden Merkmale des Stoffwechsels und der Selbstreplikation, nicht in viel einfacheren, vorhersagbaren Systemen implementiert und betrieben werden könnte. Solche minimierten Systeme würden vermutlich effizientere Maschinen für die Umwandlung und Konservierung von Energie in Brennund Wertstoffen, oder auch die Produktion von Medikamenten und intelligenten Biomaterialien darstellen als herkömmliche Wirtsorganismen wie Mikroben. Dies ist die zugrundeliegende Hypothese vieler Forschungsansätze, die unter dem Begriff der „Minimalzelle“ zusammengefasst sind.

Die Suche nach minimalen Zellen, die eine Effizienzmaximierung in biotechnologischen Prozessen ermöglichen, steht daher seit vielen Jahren im Vordergrund der syntheti- schen Biologie. Teams wie die VenterGruppe, die über völlig neue Möglichkeiten der DNA-Synthese verfügen ${ }^{[1]}$ haben bereits große Erfolge dahingehend erzielt, die minimale Zahl an essentiellen Genen durch TopDown-Gen-Knockout zu reduzieren. Dadurch konnte beispielsweise das gesamte, etwa um die Hälfte an Genen reduzierte Genom eines Mikroorganismus konstruiert werden, das in der Lage ist, die essentiellen Funktionen der ursprünglichen Zelle nahezu vollständig zu übernehmen.

Obwohl diese Studien es grundsätzlich ermöglichen können, ein minimalisierte Wirtszelle zu entwickeln, sind sie aufgrund ihrer Vorgehensweise nicht dazu geeignet, einen hinreichenden Satz an Funktionselementen zu definieren, der zum Aufbau eines
[*] Prof. Dr. P. Schwille, Dr. H. Mutschler Zelluläre und molekulare Biophysik MPI für Biochemie

Am Klopferspitz 18, 82152 Martinsried (Deutschland)

E-Mail: schwille@biochem.mpg.de

Prof. Dr. J. Spatz

MPI für medizinische Forschung

Jahnstraße 29,69120 Heidelberg (Deutschland)

Prof. Dr. K. Landfester, Dr. S. Wegner

MPI für Polymerforschung

Ackermannweg 10, 55128 Mainz (Deutschland)

Prof. Dr. E. Bodenschatz, Prof. Dr. S. Herminghaus

MPI für Dynamik und Selbstorganisation

Am Fassberg 17, 37077 Göttingen (Deutschland)

Prof. Dr. V. Sourjik, Dr. T. J. Erb

MPI für terrestrische Mikrobiologie

Karl-von-Frisch-Str. 16, 35043 Marburg (Deutschland)

Prof. Dr. P. Bastiaens, Dr. P. Bieling

MPI für molekulare Physiologie

Otto-Hahn-Str. 11, 44227 Dortmund (Deutschland)

Prof. Dr. R. Lipowsky, Dr. R. Dimova, Dr. T. Robinson MPI für Kolloide und Grenzflächen

Wissenschaftspark Golm, 14424 Potsdam (Deutschland)

Prof. Dr. A. Hyman, Dr. T.-Y. D. Tang

MPI für molekulare Zellbiologie und Genetik

Pfotenhauerstraße 108, 01307 Dresden (Deutschland)

Prof. Dr. P. Dabrock

Friedrich-Alexander-Universität Erlangen-Nürnberg

Fachbereich Theologie

Kochstraße 6, 91054 Erlangen (Deutschland)

Dr. J.-C. Baret

University of Bordeaux - Centre de Recherches Paul Pascal

115 Avenue Schweitze, 33600 Pessac (Frankreich)

Dr. T. Vidakovic-Koch, Prof. Dr. K. Sundmacher

MPI für Dynamik komplexer technischer Systeme Sandtorstraße 1, 39106 Magdeburg (Deutschland)

E-Mail: sundmacher@mpi-magdeburg.mpg.de

(D) Die Identifikationsnummern (ORCIDs) der Autoren sind unter https://doi.org/10.1002/ange.201802288 zu finden. 
lebenden Systems von Grund auf erforderlich wäre. Ein solcher De-novo-Ansatz findet sich eher in intellektueller Nachbarschaft des „Origin-of-Life“-Feldes, wo es darum geht, die Schlüsselkomponenten einer historisch plausiblen Protozelle zu identifizieren. Dementsprechend wurden viele Arbeiten zur Bildung, zum Wachstum und zur Teilung von Membranvesikeln, ${ }^{[2]}$ zur Replikation von Nukleinsäuren in Protozellen $^{[3]}$ und zur primitiven Biokatalyse ${ }^{[4]}$ von „Originof-Life"-Forschern vorangetrieben, die sich notwendigerweise eines solchen „Bottom-up“-Ansatzes bedienen müssen.

Die grundlegenden Frage, die den Kern aller Lebenswissenschaften betrifft, nämlich was Leben eigentlich ausmacht und wie es in einem minimalen System rekonstruiert werden könnte, werden in der aktuellen Forschung zur synthetischen Biologie derzeit nur am Rande behandelt. Obwohl Protozellen grundsätzlich in allen Definitionen der synthetischen Biologie enthalten sind, gibt es bisher nur wenig aktive Forschung auf diesem Gebiet. Ursache als auch Konsequenz war dabei der vergleichsweise langsame Fortschritt der Protozellforschung in den letzten zehn Jahren, ganz im Gegensatz zu synthetisch-biologischen Top-down-Ansätzen, die die Entwicklung fortgeschrittener genetischer Schaltkreise durch Gentechnik in Organismen auf Systemebene vorantrieben.

Das ist sicherlich darauf zurückzuführen, dass biologische Systeme wie fast alle autonomen Systeme generell sehr viel leichter manipuliert als grundsätzlich verstanden werden können. Die bisherige Unterrepräsentierung des „Bottomup"-Ansatzes ist auch die Folge eines bis vor kurzem nur mäßigen Interesses an der synthetischen Biologie seitens anderer naturwissenschaftlicher Disziplinen wie der Chemie und Physik. Obwohl an der Schnittstelle zwischen Chemie und Biologie sowie Physik und Biologie in den letzten Jahrzehnten enorme Fortschritte erzielt wurden, haben sich bislang nur einzelne Gruppen weltweit daran versucht, wesentliche Merkmale lebender Systeme von unten nach oben zu rekonstruieren, während die überwiegende Mehrzahl größerer Konsortien derzeit vor allem eine anwendungsorientierte synthetische Biotechnologie anstrebt. Unsere kürzlich gegründete MaxSynBio-Initiative kann also durchaus als Vorreiter einer überfälligen Entwicklung verstanden werden.

In MaxSynBio werden wir die synthetische Biologie aus der Perspektive der Grundlagenforschung betrachten. Unser primäres Ziel ist eine echte „Bottom-up“-Synthese hin zu minimalen lebenden Systemen durch sukzessive Konstruktion komplexer Funktionalitäten aus gut charakterisierten funktionellen Molekülen, Netzwerken und Modulen. In diesem Kurzaufsatz werden wir die vielfältigen Aufgaben und Aspekte unserer Forschungsinitiative diskutieren, die derzeit als 6-Jahres-Projekt durchgeführt wird, sich aber sicherlich auf einen viel größeren Zeitrahmen erstrecken muss, um ihre Ziele zu erreichen. Wir werden auch kurz auf den Stand der Technik in den einzelnen Forschungszielen eingehen, von denen viele derzeit von anderen Gruppen und Konsortien weltweit verfolgt werden.

\section{Wie und was wird von unten nach oben entwickelt?}

Lebende Organismen sind komplexe, sich selbst organisierende Systeme mit folgenden wichtigen Eigenschaften:

Sie bestehen aus Teilsystemen.

Sie tauschen ständig Materie und Energie mit ihrer Umgebung aus.

Sie organisieren sich selbst und regulieren ihre raumzeitlichen Eigenschaften.

Sie können sich selbstständig bewegen, wachsen und sind entwicklungsfähig.

Sie sind fortpflanzungsfähig.

Sie reagieren auf und kommunizieren mit ihrer Umgebung.

Um die Komplexität der zu untersuchenden Objekte handhabbar zu halten, zielen wir in erster Linie nicht auf die Rekonstruktion einer gesamten synthetischen Zelle ab. Stattdessen ist unsere Forschung anhand der folgenden Liste von Kriterien bzw. Teilprozessen strukturiert, die unserer Ansicht nach für die Existenz lebender Zellen konstitutiv sind:

Energieversorgung: Alle aktiven Prozesse in lebenden Systemen benötigen eine kontinuierliche Versorgung mit Energie und Materialien, entweder aus der extrazellulären Umgebung oder aus anderen Teilsystemen. In vielen Fällen ist die Energieversorgung und -speicherung eng mit dem Zellstoffwechsel verbunden, d.h. mit der enzymatisch gesteuerten Umwandlung von Energie in chemische Substanzen, die für bestimmte Prozesse und Subsysteme benötigt werden, oder mit der Umwandlung von Nährstoffen in leicht verfügbare Energiekomponenten, die zur Erfüllung zellulärer Funktionen benötigt werden.

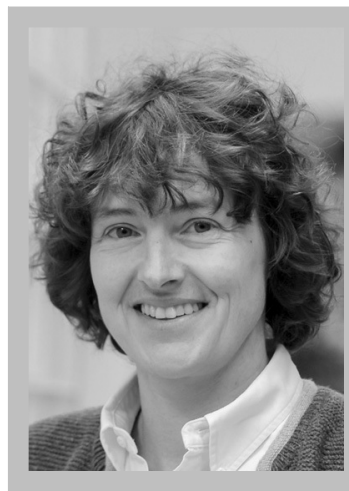

Petra Schwille promovierte 1996 bei Manfred Eigen am MPI für Biophysikalische Chemie in Göttingen. Nach einem Postdoktorat an der Cornell University (Ithaca, NY) gründete sie 1999 eine eigene Forschungsgruppe am MPI Göttingen und nahm 2002 eine Professur am Lehrstuhl für Biophysik am BIOTEC der TU Dresden an. 2011 wurde sie zum Mitglied der Max-Planck-Gesellschaft und zur Direktorin des MPI für Biochemie, Martinsried, ernannt. Ihre Forschung reicht von der Einzelmolekül-Biophysik bis hin zur synthetischen Biologie künstlicher Zellen.

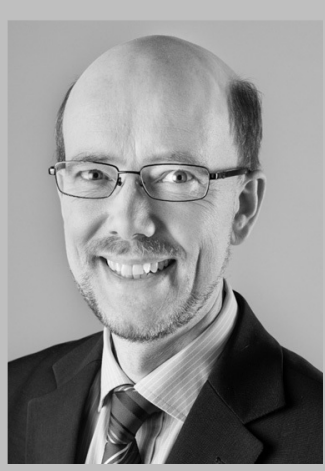

Kai Sundmacher promovierte 1995 an der Technischen Universität Clausthal. 1999 wurde er Professor für Systemverfahrenstechnik an der Otto-von-Guericke-Universität Magdeburg. Seit 2001 ist er Direktor und wissenschaftliches Mitglied des MPI für Dynamik komplexer technischer Systeme in Magdeburg. Seine Forschungsinteressen gelten der Entwicklung nachhaltiger Produktionssysteme in der Chemie und Biotechnologie, erneuerbaren Energiesystemen und synthetischen Biosystemen. 
Stoffwechsel: Stoffwechselprozesse sind das Markenzeichen des Lebens. Neben ihrer grundsätzlichen Bedeutung sind sie von zentraler Bedeutung für industrielle Produktionsprozesse. Metabolische Reaktionskaskaden und Netzwerke in biologischen Zellen sind von beeindruckender Komplexität. In MaxSynBio wollen wir eine voll funktionsfähige Stoffwechselkaskade rekonstruieren und gleichzeitig deren Komplexität auf ein Minimum reduzieren. Als Beweis des Prinzips, aber auch um eine praktische Anwendung zu demonstrieren, konzentrieren wir uns auf den CETCH-Zyklus, einen synthetischen Reaktionsweg, der $\mathrm{CO}_{2}$ abtrennt und in organische Verbindungen umwandelt.

Wachstum: Der Begriff Wachstum wird hier im Zusammenhang mit der Zellentwicklung verwendet, d. h. er bezieht sich vor allem auf die Volumenzunahme einer einzelnen Zelle. Das Zellwachstum kann entweder durch allmähliche Gewinnung von Material aus dem extrazellulären Medium oder durch Fusion erfolgen. Das Wachstum geht oft der Zellteilung voraus. Wir betrachten beide Prozesse als Schlüsselphänomene proliferierender Zellen.

Replikation und Division: Eine Mutterzelle teilt sich und produziert zwei Tochterzellen. Bevor eine Teilung erfolgen kann, müssen die in den Chromosomen gespeicherten genomischen Informationen repliziert und das duplizierte Genom zwischen den Zellen aufgeteilt werden. Generell gilt die Selbstreplikation eines Informationsträgers als der wichtigste ursächliche Prozess, der für die Entstehung von Leben notwendig ist. In einer synthetischen Zelle kann dieser Informationsträger DNA sein, die mithilfe von Polymerasen repliziert wird. Alternativ könnte auch ein selbstreplizierendes RNA-basiertes System für einfachere zelluläre Designs verwendet werden. Eine Art von Teilungsmechanismus ist die binäre Spaltung, bei der das Erbgut zu gleichen Teilen in zwei Tochterzellen zerlegt wird. Um sich zu teilen, muss eine Zelle daher polarisiert, d.h. räumlich geordnet werden. Die Zellpolarität kann auf Unterschieden in Form, Struktur und Funktion von Zellen basieren. Fast alle Zelltypen bilden Polaritätsmuster, die es ihnen ermöglichen, spezialisierte Funktionen räumlich zu trennen.

Signalïbertragung und Motilität: Neben der Kontrolle des Zellwachstums und der Differenzierung ist die Morphogenese einer der grundlegenden Aspekte der Biologie. Sie bewirkt, dass eine Zelle sich polarisiert oder ein Organismus seine Form entwickelt. Morphogenetische Reaktionen können durch Chemikalien oder mechanische Signale aus der Umwelt sowie durch Zell-Zell-Interaktionen hervorgerufen werden. Diese Reize lösen eine spontane und aktive Reaktion der Zellen aus, z.B. Adhäsion an Oberflächen, gerichtete Bewegung innerhalb ihrer Umgebung oder Zytoskelettorganisation, die wiederum die Teilung beeinflusst.

Im Rahmen von MaxSynBio fassen wir die Gesamtheit der oben genannten Lebensvorgänge unter dem Begriff „minimales Zellsystem“ zusammen. Die Kombination dieser Prozesse ist die Voraussetzung für ein funktionierendes Lebewesen. Das minimale Zellsystem basiert auf allen oben genannten Schlüsselaspekten, wobei die sehr wichtige Ausgangsbedingung der Kompartimentierung bzw. räumlichen Unterteilung gegeben ist. Fast alle Lebensprozesse finden in Kompartimenten (oder Subkompartimenten) statt, die aus
Membranstrukturen bestehen, die wiederum durch eingebettete Proteine funktionalisiert sind. Dieser Kompartimentierung verdanken es lebende Systeme unter anderem, dass sie weit abseits des thermodynamischen Gleichgewichts arbeiten können. Aus diesem Grund müssen auch synthetische Lebensprozesse in zellähnlichen Mikrokompartimenten rekonstituiert werden.

Grundsätzlich gibt es keine Einschränkungen hinsichtlich der zu verwendenden Moleküle und Materialien. Im Hinblick auf die Art der Funktionselemente, die die geforderten komplexen Eigenschaften überhaupt umsetzen könnten, bietet es sich jedoch an, zunächst auf bereits bekannte Biomoleküle, und hier vor allem Proteine, zu setzen. Die effiziente Erzeugung und Rekonstitution von funktionellen Proteinen in zellfreien Umgebungen ist daher von größter Bedeutung und wahrscheinlich die größte Herausforderung für Unternehmungen wie dieser. Allerdings muss jeder zuküntige Engineering-Ansatz auch mögliche nicht-natürliche Analoga von Funktionselementen in Betracht ziehen. Darüber hinaus müssen intelligente neue Laborroutinen eingesetzt werden, um flüssigkeitsbasierte Systeme im Maßstab von Zellen zu assemblieren. Die Bedeutung neuer nano- und mikrofluidischer Handhabungsroutinen in der Bottom-upsynthetischen Biologie kann daher nicht überschätzt werden. Wir werden kurz auf diese Aspekte eingehen und erläutern, wie wir sie in unserer Initiative einsetzen werden (Abbildung 1).

Es liegt auf der Hand, dass nur ein wirklich interdisziplinäres Forscherteam versuchen kann, all diese Aufgaben gemeinsam zu lösen. Wichtig ist, dass eine solche synthetische Herangehensweise an biologische und biomimetische Systeme nicht nur eine technische Herausforderung darstellt, sondern auch der Öffentlichkeit angemessen vermittelt werden muss, da sie auf verschiedenen Reflexionsebenen mit gerechtfertigten und ungerechtfertigten Ängsten oder starken ethischen Bedenken konfrontiert sein kann. Zu unserem Konsortium gehören daher auch Partner aus den Geisteswissenschaften, die unsere wissenschaftliche Arbeit bewusst und mit entsprechenden ethischen Überlegungen begleiten.

Im Folgenden skizzieren wir kurz, welche besonderen Ziele und Probleme im Allgemeinen angegangen werden müssen, und wie wir sie anzugehen gedenken.

\subsection{Energieversorgung}

Ein einzigartiges Merkmal lebender Zellen ist die Fähigkeit, Energie aus ihrer Umgebung zu gewinnen und diese Energie für Aktivitäten wie Wachstum, Bewegung oder Fortpflanzung zu nutzen. Im Allgemeinen wird die Energie von Nährstoffen (in der Zellatmung) oder Licht (in der Photosynthese) über Atmungs- oder photosynthetische Elektronentransferketten in einen Protonengradienten über die Zellmembran umgewandelt, der schließlich für die Synthese von Adenosintriphosphat (ATP) genutzt wird. Um lebensähnliche Prozesse in synthetischen Zellen aufrechtzuerhalten, ist eine kontinuierliche Energiezufuhr erforderlich. Daher ist es unser Ziel, Energierückgewinnungsmodule zu entwerfen und zu bauen, die dazu bestimmt sind, einer 
Natural Cell

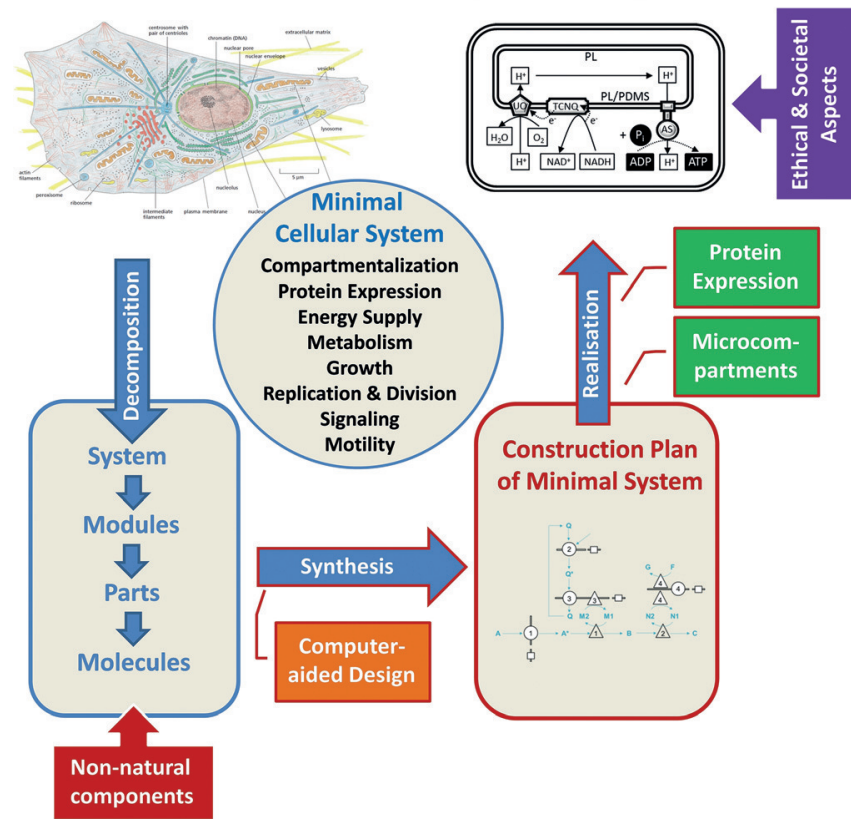

Abbildung 1. In MaxSynBio werden wesentliche Merkmale und Prozesse eines so genannten "minimalen Zellsystems" (Kompartimentierung, Proteinexpression, Energieumwandlung, Stoffwechsel, Wachstum, Replikation und Teilung, Signalisierung und Motilität) zum besseren Verständnis natürlicher Zellen synthetisiert. Die Systemsynthese basiert auf natürlichen und nicht-natürlichen Komponenten und sollte langfristig durch computergestützte Entwurfswerkzeuge unterstützt werden, die minimale Systemdesigns ermöglichen. Die Realisierung der Entwürfe wird durch spezielle Technologieplattformen (Proteinexpression, Mikrofluidik) unterstützt. Der gesamte Bottom-up-Workflow der synthetischen Biologie wird ferner unter ethischen und gesellschaftlichen Gesichtspunkten betrachtet und bewertet.

künstlichen Zelle kontinuierlich Energie in Form von ATP zuzuführen.

In der Natur ist die ATP-Regeneration an NikotinamidKofaktoren $(\mathrm{NAD}(\mathrm{H})$ oder NADP $(\mathrm{H})$ ) gekoppelt. In manchen Archaeen können diese Prozesse jedoch entkoppelt werden, wenn der für die ATP-Synthese notwendige Protonengradient durch die lichtgetriebene Protonenpumpe Bakteriorhodopsin (BR) erzeugt wird. Bisher wurden nur wenige Versuche zur Nachahmung der Energieregeneration unter Invitro-Bedingungen berichtet. ${ }^{[5]}$ Die meisten von ihnen konzentrieren sich auf die Umwandlung von Lichtenergie in ATP. ${ }^{[5 a-c]}$ Die Kombination von ATP-Synthase (ATPase) und BR erregte dabei große Aufmerksamkeit. ${ }^{[5 b]}$ Vor kurzem wurde eine Kombination aus Photosystem II, einem Proteinkomplex, der in der Lage ist, Wasser photokatalytisch zu spalten, und ATPase für die lichtbetriebene ATP-Regeneration gezeigt. Die chemische Energieumwandlung in ATP wurde dagegen weniger untersucht. ${ }^{[5 \mathrm{~d}]}$ Bisher konnte kein synthetisches System nachgewiesen werden, das in der Lage ist, aus einem importierten Substrat (z.B. Glukose) chemische Energie umzuwandeln, das mit der ATP-Regeneration über Nikotinamid-Kofaktoren-Recycling gekoppelt ist. Nicotinamid-Kofaktoren sind die häufigsten Redox-Kofaktoren in lebenden Systemen. Sie sind an vielen enzymatischen
Umwandlungen beteiligt, und ihr Recycling ist von hoher Praxisrelevanz.

Die Literaturbeispiele zeigen bereits, dass das Thema Energieversorgung in synthetischen Bottom-up-Systemen weitgehend ungelöst ist. Die Assemblierung von synthetischen Energieumwandlungssystemen aus biologischen und chemischen Komponenten ist eine Aufgabe von erheblicher Komplexität. Um sie zu bewältigen, sind intelligente Vereinfachungen erforderlich. Unser Ziel ist es, solche Strategien für die Generierung von Funktionsteilen und deren Integration in Funktionsmodule zu entwickeln. Unser funktioneller Ansatz besteht aus einem Membranprotein oder einem chemischen Katalysator, der in einen geeigneten Container (ein Membrankompartiment oder ein Hybridvesikel aus Polymeren) eingebettet ist. Als ersten Schritt in diese Richtung haben wir eine mikrofluidische Hochdurchsatzmethode adaptiert, um stabile, definierte Liposomen, sogenannte tröpfchenstabilisierte riesige unilamellare Vesikel (dsGUVs) zu erzeugen und mit ATPase zu funktionalisieren. ${ }^{[6]}$ Das Anlegen eines $\mathrm{pH}$-Gradienten bewirkt hier dann idealerweise die Produktion von ATP. Weiterhin wurde ein komplexes, chemisch energiebetriebenes ATP-Regenerationsfunktionsmodul, ein künstliches Mitochondrium, konzipiert. $^{[7]}$

\subsection{Stoffwechsel}

Der Stoffwechsel ist die dynamische Chemie der Zellen, die Energie und Bausteine für die drei- und vierdimensionale Selbstorganisation des Lebens liefert. In der Zelle ist der Stoffwechsel als komplexes Zusammenspiel von Katalysatoren („Enzymen“) und Substraten („Metaboliten“) organisiert. Langfristiges Ziel von MaxSynBio ist es, komplexe Stoffwechselnetzwerke innerhalb definierter Kompartimente aufzubauen, die mit bekannten Energiemodulen gekoppelt werden können. Aus anwendungstechnischer Sicht ist der Aufbau von Multi-Enzym-Kaskaden in Kompartimenten ein attraktives Zwischenziel auf dem Weg zu minimalen „Zellfabriken" für die maßgeschneiderte Herstellung von erwünschten Verbindungen. Solche mehrstufigen Prozessschritte könnten als Alternative zu herkömmlichen Topdown-Bemühungen in der Biotechnologie dienen, wie z.B. dem „Metabolic Engineering“ von Mikroorganismen.

Unsere Bemühungen, minimale Stoffwechselkompartimente zu schaffen, werden durch jüngste Entwicklungen befördert. Einerseits wurde bereits gezeigt, dass Enzyme und chemische Reaktionsnetzwerke im definierten Raum von Polymeren und Vesikeln eingebracht, kontrolliert und funktionell gehalten werden können. ${ }^{[6,8]}$ Andererseits ist auch der Aufbau komplexer Reaktionsnetzwerke zur Herstellung von erwünschten Verbindungen in vitro prinzipiell möglich. Bemerkenswerte Beispiele sind die Verwendung komplexer Enzymkaskaden in vitro zur Herstellung von Monoterpenen, Isobutanol bzw. Polyhydroxybutyrat aus Glukose als Ausgangsstoff. ${ }^{[9]}$

Während es in jüngster Zeit Fortschritte bei der Rekonstruktion und Kontrolle komplexer Enzymnetzwerke in vitro gab, konzentrierten sich die meisten Ansätze bisher auf die Nutzung natürlich vorhandener Reaktionskaskaden. Das 
volle Potenzial der synthetischen Biologie kann jedoch erst dann ausgeschöpft werden, wenn es möglich wird, maßgeschneiderte nichtnatürliche Reaktionsnetzwerke rationell zusammenzustellen. In einem „Proof-of-Principle“ haben wir kürzlich einen synthetischen Weg zur Abscheidung und Umwandlung von Kohlendioxid entwickelt und realisiert. Der so genannte CETCH (Crotonyl-Coenzym A (CoA)/ Ethylmalonyl-CoA/Hydroxybutyryl-CoA)-Zyklus ist ein metabolisches In-vitro-Netzwerk von 17 Reaktionen, das mit Enzymen aus neun verschiedenen Organismen aufgebaut wurde ${ }^{[10]}$ Während es möglich war, eine einfache Version des CETCH-Zyklus durch das Konzept der metabolischen Retrosynthese zu entwerfen und zusammenzustellen, ${ }^{[1]}$ war ein robuster Betrieb des Systems erst nach mehreren Optimierungsrunden möglich, die das Enzym(re-)design und das Prinzip des metabolischen Korrekturlesens umfassten. ${ }^{[11,12]}$ In seiner Version 5.4 ist der künstliche $\mathrm{CO}_{2}$-Fixierungszyklus etwas schneller und benötigt $20 \%$ weniger Energie pro fixiertem $\mathrm{CO}_{2}$ als der natürliche Calvin-Zyklus, auf dem die Photosynthese beruht.

Die nächsten Anstrengungen konzentrieren sich auf die weitere Optimierung des CETCH-Zyklus mit modellbasierten Ansätzen, wie sie aus der Verfahrenstechnik bekannt sind, sowie auf die Kopplung des künstlichen Reaktionsnetzes an Energie- (und Kofaktor-) Regenerationsmodule, ${ }^{[7]}$ um dessen kontinuierlichen Betrieb zu ermöglichen. Insgesamt zielen diese Ansätze darauf $a b$, eine synthetische Alternative zur photosynthetischen $\mathrm{CO}_{2}$-Fixierung $\mathrm{zu}$ etablieren, um das Treibhausgas $\mathrm{CO}_{2}$ als zukünftigen Kohlenstoff-Rohstoff für eine nachhaltige, kohlenstoffarme Bio-Wirtschaft zu nutzen.

\subsection{Wachstum}

Das Wachstum der protozellulären Kompartimente kann entweder durch Fusion oder durch allmähliche Materialgewinnung von außen erfolgen. Neben der reinen Volumenvergrößerung liefert das Wachstum neue Energie, Informationen und Nährstoffe für die Entwicklung der Zellen. Im Rahmen von MaxSynBio entwickeln wir verschiedene Strategien für das Wachstum von Tröpfchen, Liposomen und Polymeren als vollsynthetische Analoga (Abbildung 2).

Vesikel stellen eine relativ enge Annäherung an lebende Zellen bzw. deren Organellen dar. Die bahnbrechenden Studien zum Vesikelwachstum ${ }^{[13]}$ stützten sich hauptsächlich auf Fettsäuremizellen und Vesikel, die als Protozellmodelle umfassend untersucht wurden. Die spontane Aufnahme von Fettsäuren in vorgeformte Vesikel wurde oft als primitiver Wachstumsmechanismus angesehen. Während jedoch Fettsäuren effizient eingebaut werden, sind Phospholipide, die Hauptbestandteile moderner Zellmembranen, hochgradig unlöslich, und dieser Wachstumsmechanismus ist nicht anwendbar. Auch der Einbau von Membranproteinen in Fettsäure-Doppelschichten sowie die Vollsynthese von Proteinen in speziellen Einzelfällen wurde erreicht.

Die kontinuierliche Suche nach realistischen Protozellmodellen auf Basis natürlicher Phospholipide hat dazu geführt, dass Phospholipide in der Regel von außen zugeführt werden. Das Vesikelwachstum wird z. B. durch Zugabe eines

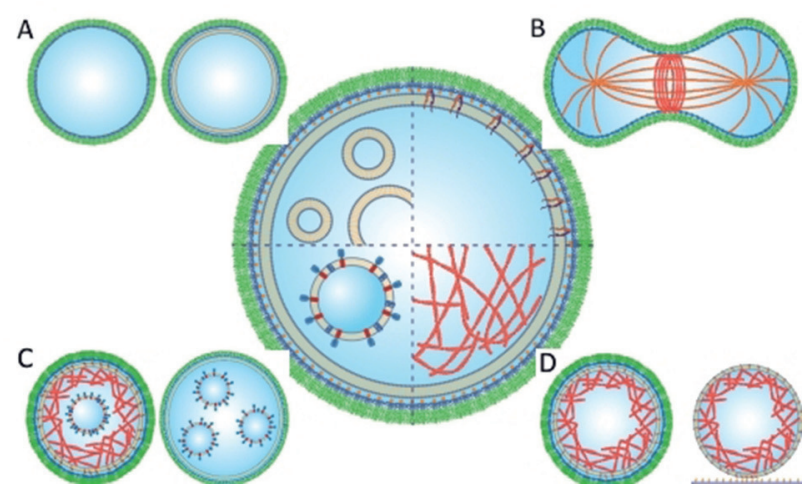

Abbildung 2. Schematische Darstellungen des modularen Engineering Ansatzes für die Bottom-up-Assemblierung von zellartigen Kompartimenten, die A) wachsen können, B) sich teilen, C) Energie und Kräfte erzeugen und D) zelluläre Funktionen wie Adhäsion und Migration erfüllen.

kationischen Vorläufers, der durch einen in die Membran eingebetteten Katalysator zu einem Membranlipid hydrolysiert wird, nachgewiesen. ${ }^{[14]}$ Diese recht exotische Chemie der Membranlipide kann jedoch Wechselwirkungen von und mit anderen biologischen Spezies (z. B. Membranproteinen) behindern. Bei authentischen Phospholipiden wurde die Vesikelvergrößerung daher auch durch eine physikalische Vesikelfusion erreicht, die durch verschiedene Auslöser initiiert werden kann. ${ }^{[15]}$ So könnte die Membranfusion als einfacher Wachstumsmechanismus nicht nur zur Vergrößerung der Membranfläche, sondern auch zur Versorgung anderer notwendiger chemischer Spezies eingesetzt werden.

Unser vorrangiges Ziel ist es, eine dynamische und kontrollierte Volumenvergrößerung der Mikrokompartimente zu erreichen. Die verschiedenen möglichen Modellcontainer auf Basis von Fettsäuren, Phospholipiden oder Polymeren erfordern konzeptionell unterschiedliche Ansätze, sind aber alle weitgehend auf Konzentrationsgradienten als treibende Kräfte, elektrostatische Wechselwirkungen und Licht als äuBere Reize, sowie deren Kombination, angewiesen. Das kontrollierte Wachstum von Tröpfchen kann durch Koaleszenz von zwei Tröpfchen mittels mikrofluidischer oder optischer Manipulation erreicht werden. Bei Vesikeln setzen wir auf osmotische, elektrostatische und andere Reize, die durch Membranspannung moduliert werden. Das Wachstum von Polymerisaten erfolgt anhand responsiver Nanopartikel, die amphiphiles Material enthalten, das bei Anwendung eines Reizes freigesetzt und in die Polymermembran integriert wird. Letzteres führt dann zum Größenwachstum der Vesikel.

\subsection{Replikation und Division}

Teilung und Replikation sind die offensichtlichsten und markantesten Merkmale lebender Zellen. Diese beiden Hauptaspekte sind funktional getrennt, müssen aber für die erfolgreiche Generierung von Nachwuchs aufeinander abgestimmt werden: in eine dramatische mechanische Transformation, die zur vollständigen Aufspaltung eines Kompartiments führt, und eine Replikation des Genoms, dessen Ko- 
pien adäquat auf die beiden neuen Kompartimente verteilt werden müssen. In modernen Zellen widmen sich große Proteinmaschinerien der räumlichen Organisation und Orchestrierung dieser Prozesse. Die vollständige Rekonstitution eines replizierenden und sich teilenden Membranraums mit genomischer DNA bleibt somit eine große Herausforderung für die Herstellung einer künstlichen Protozelle. Darüber hinaus erfordert die Selbstreplikation einer lebenden Zelle auf Proteinbasis die Replikation und Synthese von mehr als hundert aktiven Genen, die für die Translation und Ribosomenbiogenese benötigt werden ${ }^{[16]}$ - eine monumentale Aufgabe, deren Erfüllung noch weit entfernt ist. Die Replikation und Teilung von semiautonomen zellulären Designs konnte jedoch in vitro durch translationsgekoppelte Replikation von Minimalgenomen auf DNA-Basis erreicht werden. RNA ist ein attraktiver alternativer Informationsträger, der die Selbstreplikation und sogar die Evolution in zellähnlichen Systemen unterstützen kann. ${ }^{[17]}$ Es bleibt zu erforschen, wie solche selbstreplizierten RNA-Moleküle zuverlässig in den Tochterzellen getrennt werden können.

Spannende Arbeiten im Sinne einer Kopplung von Kompartimentwachstum und -teilung wurden mit von Fettsäurevesikeln umschlossener RNA durchgeführt. ${ }^{[2 b]}$ Aufgrund ihrer relativ geringen Stabilität und „Undichtigkeit“ haben Fettsäurevesikel jedoch nur ein begrenztes Potenzial für die Implementierung anspruchsvollerer funktionaler (Protein-)Module, und Vesikel aus Phospholipiden bieten eine deutlich größere funktionelle Variabilität.

In den letzten Jahren hat man erkannt, dass biochemische Makromoleküle im Zellinneren auch membranlose Kompartimente (Tröpfchen) durch Flüssigphasen-Entmischung bilden können. ${ }^{[18]}$ Beispiele hierfür sind die Proteine PGL-3 und FUS, ${ }^{[19]}$ die in Lösung spontan zu tropfenförmigen Kompartimenten kondensieren und sich auf diese Weise von ihrer Umgebung abtrennen. Diese Tröpfchen können durch ständige Aufnahme von Material aus der Umgebung wachsen, und es gibt Hinweise, dass sie sich unter bestimmten Umständen auch teilen. Damit stellen sie ein weiteres vielversprechendes Konzept für den Bau eines künstlichen minimalen Zellsystems dar. ${ }^{[20]}$

Im Hinblick auf die mechanische Umwandlung von Phospholipidkompartimenten wurde in den letzten Jahren viel an der Rekrutierung von lokal membrantransformierenden Proteinhüllen und -maschinerien auf Riesenvesikeln (GUVs) gearbeitet, ${ }^{[21]}$ während die Rekonstitution von Proteinen, die aktiv an der Zellteilung beteiligt sind, sich bislang als sehr kompliziert erweist. Daher steht auch die Aufgabe einer vollständigen und autonomen proteininduzierten Teilung von Vesikeln auf zellulären Skalen noch weit am Anfang, wobei wichtige Proteinfaktoren hierfür bereits identifiziert wurden. Von besonderem Interesse sind die bekannten eukaryotischen und prokaryotischen Teilungsmaschinerien auf Basis von Actomyosin und FtsZ, ${ }^{[22]}$ aber auch entsprechende Systeme aus Archaeen sind in letzter Zeit in den Fokus gerückt. ${ }^{[23]}$

Im Hinblick auf eine mögliche DNA-Replikation in Membrankompartimenten konnte gezeigt werden, dass Knospenbildung und Fusion von Vesikeln aus verkapselten Polymerlösungen aus dem Zusammenspiel von entropischer
Depletion und Benetzungseffekten resultieren können. ${ }^{[14,15 b]}$ Die Auftrennung chromosomaler DNA in Vesikeln kann zumindest teilweise durch rein entropische Abstoßung erreicht werden. ${ }^{[24]}$ Alternativ können spindelförmige Strukturen, die eine aktive Trennung von DNA induzieren, aus gereinigten Komponenten bakterieller Plasmid-Segregationsmaschinen rekonstruiert werden. ${ }^{[25]}$

Die laufenden Arbeiten in unserem Konsortium umfassen verschiedene Aspekte der minimalen Zellteilung und -replikation. Wir konzentrieren uns auf physikalische, insbesondere temperaturinduzierte Transformationen von Tröpfchen und beleuchten auch den Aspekt der mechanischen Transformation durch kraftübertragende Proteinmaschinen, die in die Tröpfchen eingebracht wurden. ${ }^{[26]}$ Wir kombinieren theoretische und experimentelle Maßnahmen, um zu untersuchen, wie Vesikel durch physikalische Signale, insbesondere durch Licht, mechanisch transformiert werden können und wie diese Transformation aufgrund lokaler und globaler Membraneigenschaften in eine vollständige Teilung übergehen kann. Im Mittelpunkt steht dabei auch die Charakterisierung der genauen Verteilung des Inhalts in zwei Tochterkompartimente während eines erzwungenen Teilungsprozesses. Schließlich werden wir die Chromosomenreplikation in vitro rekonstruieren und an den mechanischen Prozess der Kompartiment-Teilung koppeln.

\subsection{Signalübertragung und Motilität}

Obwohl die Rekonstitution und grundlegende Charakterisierung der zellulären Signalübertragung in minimalen Systemen ein wichtiges Forschungsziel ist, gab es aufgrund der enormen Komplexität dieser Prozesse in den letzten Jahren wenig Fortschritte. Die Geometrie biologischer Membranen ist dabei eng mit der Signalverarbeitungsfähigkeit einer Zelle verknüpft. ${ }^{[27]}$ Die Plasmamembran stellt eine Oberfläche dar, an der bei der Signalisierung durch die Rekrutierung von zytosolischen Effektoren, z.B. an membrangebundene GTPasen, eine Reduktion der Reaktionsdimensionen und damit eine Beschleunigung der Reaktion erfolgen kann. ${ }^{[28]}$ Die lokalen geometrischen Eigenschaften einer Membran beeinflussen aber auch die Fähigkeit, diese zytosolischen Effektoren überhaupt zu rekrutieren. So wird die Signalisierung von GTPasen durch die Verformung der Plasmamembran durch das Zytoskelett beeinflusst. Das Zytoskelettwachstum, das die Plasmamembran deformiert, wird wiederum von zytoplasmatischen Signalgradienten vorgegeben, die von den rekrutierten enzymatischen Effektoren ausgehen. ${ }^{[29]}$ Durch diese Rückkopplungen bzw. rekursiven Abhängigkeiten in der Triade von Membrangeometrie, Zytoskelettdynamik und Signaltransduktion kommt es zur Selbstorganisation der Informationsverarbeitung an zellulären Membranen, die kontextabhängige morphogene Reaktionen ermöglichen. ${ }^{[27,30]}$

In unseren bisherigen Arbeiten wurden GUVs mit rekonstituierten $\alpha 2 b \beta 3$-Integrinen entwickelt. Bei der Aktivierung von Integrinen durch $\mathrm{Mn}^{2+}$-Ionen reagierten die Integrine spezifisch auf eine extern präsentierte Fibrinogenmatrix, indem sie sich darauf ausbreiteten. ${ }^{[6]}$ Dieser Assay 
demonstriert, wie genau spezifische Kompartimente Signale von außen empfangen und in eine aktive Antwort des Kompartiments übersetzen können. Im nächsten Rekonstitutionsschritt soll die Kopplung von Integrinen an ein krafterzeugendes molekulares Netzwerk wie F-Actin/Myosin oder Mikrotubuli/Kinesin eine durch ein externes Signal initiierte Kompartimentmobilität bewirken.

Unser Ziel ist es, solche stigmergischen Systeme, die für das Leben auf Basis von Membranen vermutlich konstitutiv sind, synthetisch nachzustellen. Insbesondere versuchen wir, eine membranbasierende Mikroumgebung zu schaffen, die in der Lage ist, externe Signale zu empfangen, diese Signale über ihre Grenzen hinweg zu übertragen und sie in morphologische Veränderungen und Motilität des Systems zu übersetzen. Neben molekularen Netzwerken innerhalb des Kompartiments könnte die Kompartimentmobilität in einer höheren Komplexitätsebene auch durch extern angebrachte minimale Flagellen oder Wimpern erzeugt werden. Das allgemeine Konzept besteht also jeweils darin, ein synthetisches System zu rekonstruieren, das in Kombination mit spontaner oder signalgesteuerter Mobilität Signalaktivität erzeugt.

\section{Technologieplattform}

\subsection{Mikrofluidik zur Erzeugung und Manipulation von Kompartimenten}

Wie bereits skizziert, sind Tröpfchen konzeptionell das einfachste System für die Kompartimentierung von Flüssigkeiten. Tröpfchen werden leicht in zweiphasigen Systemen erzeugt, wenn eine externe Quelle genügend Arbeit liefert, um die energetischen Kosten der Erzeugung einer Tröpfchengrenzfläche zu überwinden: die Transferierung einer wässrigen Phase in Öl führt dann zur Bildung einer Dispersion. Einfache hydrodynamische Agitation führt jedoch zu einer polydispersen Verteilung der Kompartimente. Fundamentale thermodynamische Erwägungen zeigen, dass die Dispersion nur metastabil ist und spontan zu zwei durch eine minimale Oberfläche getrennten Volumenphasen zerfällt. Wenn Tröpfchen als minimale Kompartimente mit Relevanz für einen synthetischen biologischen Ansatz verwendet werden sollen, müssen daher Mittel zur Stabilisierung der Tröpfchen in einem metastabilen Zustand durch Kontrolle der Tröpfchengrößen über große Populationen entwickelt werden. Diese Aufgabe lässt sich idealerweise durch den Einsatz hochdurchsatzfähiger tröpfchenbasierter Mikrofluidik lösen. ${ }^{[31]}$

Alle in unserem Konsortium verwendeten mikrofluidischen Verfahren basieren auf der Herstellung von Poly(dimethylsiloxan)(PDMS)-Strukturen mittels Photo- und Softlithographieverfahren. ${ }^{[32]}$ PDMS ist aufgrund seines niedrigen Preises, der guten Biokompatibilität und Gasdurchlässigkeit, der hohen optischen Transparenz und der geringen Eigenfluoreszenz ein gängiges Material in der Mikrofluidik. Tröpfchen werden in einer strömungsfokussierenden Geometrie erzeugt, in der eine wässrige Phase durch eine tensidhaltige Ölphase abgeschnitten wird. Nach der Bildung werden Wasser-in-Öl-Tropfen durch Anreicherung von
Blockcopolymer-Tensiden an der Wasser-Öl-Grenzfläche stabilisiert, was zu einer Reduzierung der Ö1/Wasser-Grenzflächenspannung führt. ${ }^{[33]}$ Der Tropfendurchmesser wird hauptsächlich durch die Kanalabmessungen gesteuert, kann aber auch teilweise durch die Variation der Durchflussraten der wässrigen Phase und der Ölphase reguliert werden.

Um eine präzise Abgabe verschiedener biologischer Komponenten in vorgeformte Tröpfchen $\mathrm{zu}$ ermöglichen, können kleine und kompakte Elektroden zum Anlegen elektrischer Felder in die Mikrokanäle integriert werden. Die lokalen elektrischen Felder induzieren dann eine Destabilisierung (Poration) der Tensid-(Mono-)Schicht und ermöglichen eine kontrollierte Injektion (Pico-Injektion) der wässrigen Phase in die Tröpfchen. Das Design unserer tröpfchenbasierten Pico-Injektionseinheit wurde von Abate et al. adaptiert. ${ }^{[34]}$ Ein mikrofluidisches Durchflusskontrollsystem wird verwendet, um die Tröpfchen definiert in die Pico-Injektionseinheit einzubringen, in der sie isoliert voneinander ein elektrisches Wechselstromfeld (AC) passieren. Dieser Prozess destabilisiert die Tröpfchengrenzfläche und ermöglicht die Einbringung biologischer Reagenzien über einen Druckinjektionskanal (Abbildung 3). Das Injektionsvolumen kann über den angewendeten Druck im Injektionskanal präzise zwischen 1 und $100 \mathrm{pL}$ kontrolliert werden.

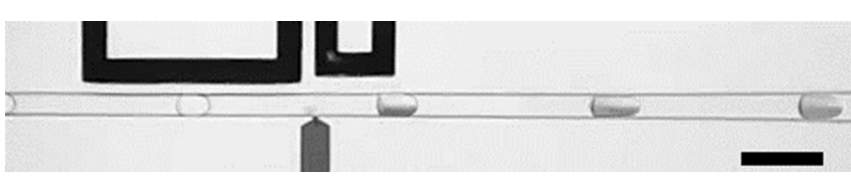

Abbildung 3. Beispiel von Pico-Injektionen (ca. $15 \mathrm{pL}$ ) einer dunklen Flüssigkeit in Tröpfchen, die den Seitenkanal passieren. Skalierung: $50 \mu \mathrm{m} .^{[6,34]}$

Neben kontrollierten Methoden zur Herstellung und Analyse von Tröpfchensuspensionen bieten mikrofluidische Systeme auch die Möglichkeit, Lipidvesikel zu handhaben. Geeignete Geometrien mit paarweisen PDMS-Pfosten nutzen die hydrodynamischen Kräfte in mikrofluidischen Kanälen aus, um einzelne oder eine kleine Zahl von GUVs zu immobilisieren. ${ }^{[35]}$ Druckgesteuerte Kammern auf dem Chip bieten eine zusätzliche Einschlussebene für die schnelle und kontrollierte Zugabe von Chemikalien, die um das GUV herum fließen, und können für parallelisierte Langzeitstudien verwendet werden. ${ }^{[36]}$

\subsection{Zellfreie Proteinproduktion}

Die Produktion von gereinigten funktionellen Proteinen ist eine Schlüsseltechnologie und leider auch ein methodischer Flaschenhals für viele Forschungsprojekte in den Lebenswissenschaften, und dies gilt auch für die hier verfolgte Bottom-up-Assemblierung von minimalen biologischen Systemen. Trotz großer Fortschritte bei der Standardisierung und Parallelisierung der Proteinaufreinigung schließen die hochspezifischen Aufreinigungsbedingungen für die verschiedenen Proteine die Entwicklung allgemein anwendbarer Methoden weitgehend aus. Geeignete Bedingungen für die 
Aufreinigung eines neuartigen Proteins können daher oft nicht durch Extrapolation von homologen Systemen identifiziert werden, und stellen insbesondere für viele der integralen und peripheren Membranproteine, die für unsere Arbeitsaufgaben entscheidend sind, nach wie vor eine große Herausforderung dar.

Eine alternative Strategie zur effizienten Integration von Proteinen in ein artifizielles System ist die zellfreie Proteinsynthese (CFPS). Hier können bakterielle und eukaryotische Zellextrakte oder rekombinante Systeme mit entsprechender RNA oder DNA versetzt werden, um Proteine direkt in vitro herzustellen. Neben der einfachen Proteinproduktion lassen sich mit CFPS auch synthetische Proteinnetzwerke aufbauen ${ }^{[37]}$ integrale und periphere Membranproteine ${ }^{[38]}$ und sogar große infektionsfähige Viruspartikel erzeugen. ${ }^{[39]}$ Bemerkenswert ist, dass in eukaryotischen Extrakten durch den Einsatz zusätzlicher mikrosomaler Membranen sogar ko- und posttranslationale Proteinmodifikationen erreicht werden konnten. ${ }^{[40]}$ Das Fehlen dieser Modifikationen ist ein wesentliches Manko in herkömmlichen bakteriellen Expressionssystemen.

In den letzten Jahren wurden CFPS-Systeme zunehmend mit Methoden der artifiziellen Bio-Kompartimentierung kombiniert. Sie eignen sich daher prinzipiell auch für die verschiedenen im MaxSynBio-Netzwerk evaluierten Mikrokompartimente wie Liposomen/Vesikel, ${ }^{[41]}$ Emulsionströpfchen, ${ }^{[42]}$ Polymere ${ }^{[43]}$ und Koazervate. ${ }^{[44]}$ Aufgrund ihrer Kompatibilität mit verschiedenen technischen Verfahren haben viele Gruppen CFPS erfolgreich bei der Entwicklung zellulärer Mimikry eingesetzt. ${ }^{[45]}$ Eine reichhaltige Literatur aus Handbüchern und Leitfäden zu den jeweiligen praktischen Herausforderungen dieser Systeme ist bereits verfügbar. ${ }^{[46]}$ Auch die neuesten Entwicklungen bezüglich des Designs von Mikrofluidiksystemen ${ }^{[47]}$ haben die Entwicklung von tröpfchenbasierten CFPS nachhaltig beschleunigt.

Innerhalb unseres Konsortiums besteht bereits ein langjähriges Interesse an der Entwicklung von tröpfchenbasierter CFPS, ${ }^{[42 b]}$ die nun zur Entwicklung neuartiger minimaler biologischer Systeme auf der Basis zellfreier Expression herangezogen wird. Die Kopplung von Transkription und Translation macht diese Methode zu einer attraktiven Wahl für die Realisierung eines teilweise oder vollständig selbstkodierten minimalen lebenden Systems. ${ }^{[48]}$ Die Schaffung eines komplexen selbstreplizierenden Systems auf Basis von CFPS bleibt jedoch ein langfristiges Ziel, da es die parallele Integration vieler verschiedener Module wie rekursive $\mathrm{Ge}$ nomreplikation, Ribosomenbiosynthese, Lipidsynthese, Teilung und Energieproduktion erfordert. Obwohl in den letzten Jahren erhebliche Fortschritte z. B. bei der De-novo-Ribosomensynthese während der CFPS erzielt wurden, ${ }^{[49]}$ sind noch erhebliche Verbesserungen der Ausbeute und Lebensdauer der CFPS-Systeme und der physikalisch-chemischen Kompatibilität der verschiedenen Module erforderlich. Diese Probleme können vermutlich durch die Optimierung der CFPS-Komponenten mithilfe von mathematischen Modellierungs- und Simulationswerkzeugen gelöst werden. ${ }^{[50]}$

\section{Design ganzer Biosysteme aus einzelnen Funktionsmodulen: eine Ingenieuraufgabe}

Für die Zukunft erwarten wir große Fortschritte auf dem Weg des Bottom-up-Designs hin zu selbstorganisierenden Biosystemen, indem die Baukästen der synthetischen Biologie kontinuierlich mit immer mehr funktionalen und gut charakterisierten Bausteinen gefüllt werden. Idealerweise werden verlässliche und gut strukturierte Bibliotheken von Molekülen, Bauteilen und Modulen geschaffen, die eine der wesentlichen Voraussetzungen für die systematische Synthese künstlicher Biosysteme sind. ${ }^{[51]}$ Auf der anderen Seite ist das computergestützte Design neuartiger Systeme basierend auf den drei beteiligten Hauptsystemskalen (Moleküle, Bauteile, Module) aus Sicht des Bioingenieurs eine große Vision. Ein solchermaßen ingenieurwissenschaftliches Vorgehen hin zum funktionalen System erfordert: a) einen Entwurf, d.h. eine klare Definition der zu erstellenden Funktionalität des Systems; b) Qualitätssicherung, d.h. die experimentelle Validierung der Funktionalitäten der Moleküle, Zugriff auf alle Teile und Module, aus denen das Zielsystem zusammengesetzt ist; c) Standardisierung, d.h. gut definierte Schnittstellen, die die funktionalen Komponenten auf jeder Ebene der Systemhierarchie stabil verbinden; und d) Massenproduktionsplattformen, d.h. Technologien, die eine reproduzierbare Manipulation und Montage aller Systemeinheiten ermöglichen. Diese vier Elemente $(\mathrm{a}-\mathrm{d})$ bilden den für die Bottomup-Produktion von Biosystemen aus funktionalen Einheiten notwendigen biotechnologischen Arbeitsablauf (workflow), analog zu den in anderen Ingenieurdisziplinen wie Verfahrenstechnik, Elektrotechnik oder Maschinenbau etablierten Workflows. ${ }^{[52]}$

Erste erfolgreiche Beispiele entlang eines computergestützten Konstruktionsweges hin zu synthetischen Zellen wurden bereits gezeigt. Schneider und Mangold ${ }^{[53]}$ postulierten den modularen Aufbau einer In-silico-Protozelle, bestehend aus einem Membranproliferationsmodul, einem Membrankontraktionsmodul und einem Positioniermodul. Theoretische Hypothesen wurden getestet, um die einzelne Module zu einem Protozellenmodell mit synchron arbeitenden Teilen zusammenzuführen. Otero-Muras und Banga ${ }^{[51]}$ schlugen einen automatisierten Entwurfsprozess vor, bestehend aus 1) einem „Forward Design“-Schritt, bei dem die Pareto-optimalen Menge synthetischer Designs für die Implementierung ermittelt wird, und 2) einem „Reverse Design"-Schritt, bei dem aus der Pareto-Menge auf mögliche Motive und konkrete Ausführungsformen von genregulatorischen Netzwerken zurückgeschlossen wird. Die Autoren illustrierten die Funktionstüchtigkeit ihres Konzepts anhand verschiedener Fallstudien, einschließlich eines Oszillatorsystems. Die beiden hier ausgewählten Publikationen zeigen, dass die Etablierung von Konstruktionskonzepten für einen systematischen Ansatz zum modularen Aufbau von Biosystemen aus Funktionsmodulen sehr hilfreich ist. In diesem Bereich erwarten wir neue spannende Entwicklungen bei der Entwicklung zellulärer Systeme mit komplexen Verhaltensweisen wie Teilung, Kognition und Motilität.

Wie von Tayar et al. ${ }^{[54]}$ diskutiert, erlauben die jüngsten Fortschritte in der zellfreien Proteinexpression die Rekon- 
struktion von Selbstorganisationsphänomenen in ReaktionsDiffusions-Systemen, insbesondere 1) Umsatzmechanismen für kontinuierliche und verlängerte Genexpressionsreaktionen, 2) programmierbare Genexpressionsreaktionen unter Verwendung biologischer Regulationselemente und 3) räumliche Verteilung und Kommunikation zwischen abgeschotteten Reaktionen. Wie die Autoren zeigen, ist die Definition von Designregeln für die Selbstassemblierung in synthetischen Biosystemen unerlässlich, um den Weg für die Realisierung autonomer selbstreplizierender Systeme zu ebnen.

Die synthetische Bottom-up-Biologie ist wie die anderen Spielarten der synthetischen Biologie natürlich nicht an die reine Reproduktion bzw. die Nachahmung der Eigenschaften und Verhaltensweisen natürlicher Systeme gebunden. Sie hat bereits damit begonnen, hybride Systeme zu generieren und $\mathrm{zu}$ erforschen, in denen natürliche mit nicht in der Natur vorkommenden Molekülen, Bauteilen oder Funktionsmodulen kombiniert werden. So haben Otrin et al. ${ }^{[7]}$ ein mit chemischer Energie betriebenes, ATP-erzeugendes künstliches Modul durch Bottom-up-Rekonstitution von ATP-Synthase und terminaler Oxidase in neuartigen Nano-Containern aus Pfropfcopolymer-Membranen und aus Hybrid-Pfropfcopolymer/Lipid-Membranen zusammengebaut. Diese Container können als vielseitiges Werkzeug für die Rekonstitution von Membranproteinen in komplexeren kompartimentierten Systemen eingesetzt werden, z.B. in Protozellen, die mit künstlichen Modulen für die Energieversorgung und für den Metabolimus ausgestattet sind. Auf diese Weise könnte die synthetische Biologie auch zur Konstruktion von Systemen beitragen, deren selbstorganisierendes Verhalten in der Natur nicht beobachtbar ist.

\section{Ontologien des Lebens und ethische Implikationen}

In den letzten Jahren wurden verschiedene Ansätze verfolgt, um mögliche gesellschaftliche Herausforderungen der bzw. innerhalb der synthetischen Biologie zu identifizieren. ${ }^{[5]}$ Die folgenden vier Aspekte sollten dabei im Umgang mit den ethischen Herausforderungen hervorgehoben werden.

Erstens müssen echte Sicherheitsprobleme identifiziert und nach Möglichkeit eliminiert werden. Ein häufig diskutiertes Thema ist das Problem des möglichen Missbrauchs von Erkenntnissen und Produkten. Da neue Informationen, Reagenzien und technologische Entwicklungen immer das Potenzial besitzen, sowohl für nützliche als auch für schädliche Zwecke genutzt zu werden, wird diese erste Herausforderung als „Dual Use Research of Concern“ (DURC) bezeichnet. Viele wissenschaftliche Organisationen haben Verhaltenskodizes als eine Art Selbstregulierungsstandards erarbeitet und umgesetzt, um die Arbeit der jeweiligen Forscher zu begleiten und zu leiten. Dennoch gibt es insbesondere im Hinblick auf den Top-down-Ansatz und auf mögliche ökologische Auswirkungen biotechnologischer Verfahren - vier Aspekte, die eine besondere und kontinuierliche Aufmerksamkeit erfordern: Erstens die Unterschiede in der Physiologie von „natürlichen“ und „synthetischen“
Organismen, zweitens die bisher unbekannte mögliche Veränderung von synthetischen Organismen in verschiedenen Lebensräumen, drittens die mögliche Evolution und Anpassung der „produzierten“ synthetischen Organismen und viertens die Möglichkeit von Mikroben, freie DNA aus der Umwelt aufzunehmen oder ihr genetisches Material mit anderen Organismen auszutauschen. Bislang sind die bestehenden Regelwerke, vor allem im Hinblick auf den Protozellansatz, jedoch so zu bewerten, dass sie die aktuellen Forschungsaktivitäten ausreichend abdecken. ${ }^{[56]}$

Zweitens, und dies ist für unseren Protozellansatz entscheidender, sind neue ethische Fragen zur möglichen Verwischung kultureller Konzepte und Distinktionen wie „Leben vs. Nicht-Leben“ oder „natürlich vs. künstlich“ entstanden und nun Gegenstand gesellschaftlicher, konzeptueller und ethischer Studien. Begriffe und Metaphern wie „Leben erschaffen“ oder „Gott spielen“ können als Versuche der Gesellschaft verstanden werden, Ausdrücke für die gegenwärtige Bedeutung und Wirkung der neuartigen technologischen Entwicklung $\mathrm{zu}$ finden. Eine Ursache für die potentielle Verunsicherung im Zusammenhang mit der synthetischen Biologie ist die Tatsache, dass der logische Wert einer Aussage wie „X gehört (nicht) zur Klasse der lebenden Systeme“ nicht nur von $X$ abhängt, sondern zum Teil auch vom jeweiligen Betrachter und seinen ontologischen und erkenntnistheoretischen Voraussetzungen. Unsere Analyse der verschiedenen Metaphern, die von Wissenschaft und Gesellschaft verwendet werden, identifiziert zwei verschiedene Prozesse, die durch die Entstehung neuer Biotechnologien verursacht werden. Einerseits kann die Leistungsfähigkeit der Biotechnologien zu tiefgreifenden Veränderungen im jeweiligen sozialen, wirtschaftlichen oder physischen Umfeld führen und somit erhebliche Auswirkungen auf die verschiedenen Lebensformen haben. Andererseits kann die Erzeugung neuartiger, nicht in der Natur vorkommender Objekte etablierte Bedeutungs- und Wertvorstellungen stören und verändern und damit Potenzial für gesellschaftliches Unbehagen schaffen.

Drittens geht die ethische und gesellschaftliche Debatte über den Umgang mit neuen Biotechnologien im Allgemeinen und der synthetischen Biologie im Besonderen dahin zu klären, wer an den Entscheidungen über die aufgeworfenen Fragen beteiligt sein muss und sollte. Es geht also nicht nur darum, ob die wissenschaftlichen Versprechen eingehalten werden, sondern auch, wie und von wem sie vorangetrieben werden. Aus diesem Grund ist die Beteiligung der Öffentlichkeit an der Wissenschaft nicht nur ein weiterer „nice to have"-Punkt auf der Agenda der Bewertung neuer Biotechnologien, sondern wird für die zukünftige Entwicklung der synthetischen Biologie entscheidend sein.

Viertens, indem man versucht, künstliches Leben aus synthetischen Chemikalien zu erschaffen, um den Prozess der Entwicklung des Lebens besser zu verstehen, stellt sich die Frage, wie solche Bemühungen grundlegende epistemologische und ontologische Konzepte wie Leben, Biodiversität oder Evolution verändern könnten. Vor diesem Hintergrund ist es eine der drängendsten Aufgaben, einen praktikablen Rahmen zu entwickeln, um die Errungenschaften der syn- 
thetischen Bottom-up-Biologie theoretisch auf ihre konzeptionellen Herausforderungen hin zu betonen. ${ }^{[57]}$

\section{G. Schlussbemerkungen}

Nach der Zelltheorie resultiert jede Zelle von einer anderen lebenden Zelle. Es wird allerdings keine Aussage getroffen, wie eine Zelle überhaupt erstmals aus dem komplizierten Zusammenspiel von nicht lebenden Bestandteilen hervorgehen könnte. Ein solcher Prozess muss jedoch in der Natur mindestens einmal stattgefunden haben und sollte daher prinzipiell auch im Labor darstellbar sein. Mit dem heutigen Wissen und unseren aktuellen (bio-)technologischen Möglichkeiten ist ein synthetischer Bottom-up Ansatz hin zu einem lebenden System aus klar definierten modularen Komponenten auf der Mikro- und Nanoskala in greifbare Nähe gerückt. Natürlich kann unser gemeinsames 6-jähriges Forschungsprojekt innerhalb von MaxSynBio nur den Beginn eines viel größeren und längeren Forschungsunternehmens markieren, das über nationale Initiativen hinausgeht und eine weitere intensive europäische und weltweite Zusammenarbeit erfordert. Die Suche nach lebenden Zellen von unten nach oben hat gerade erst begonnen.

\section{Danksagung}

Die Autoren danken für die Unterstützung ihres Forschungsnetzwerks MaxSynBio durch eine gemeinsame Förderinitiative des Bundesministeriums für Bildung und Forschung (BMBF) und der Max-Planck-Gesellschaft. Wir danken Dr. Jakob Schweizer (MPI Magdeburg) und Dr. Ilia Platzman (MPI Heidelberg) für die Unterstützung bei der Abfassung des Artikels in Wort und Bild.

\section{Interessenkonflikt}

Die Autoren erklären, dass keine Interessenkonflikte bestehen.

Zitierweise: Angew. Chem. Int. Ed. 2018, 57, 13382-13392 Angew. Chem. 2018, 130, 13566-13577

[1] a) D. G. Gibson, G. A. Benders, C. Andrews-Pfannkoch, E. A Denisova, H. Baden-Tillson, J. Zaveri, T. B. Stockwell, A. Brownley, D. W. Thomas, M. A. Algire, C. Merryman, L. Young, V. N. Noskov, J. I. Glass, J. C. Venter, C. A. Hutchison, H. O Smith, Science 2008, 319, 1215-1220; b) C. A. Hutchison 3rd, R. Y. Chuang, V. N. Noskov, N. Assad-Garcia, T. J. Deerinck, M. H. Ellisman, J. Gill, K. Kannan, B. J. Karas, L. Ma, J. F. Pelletier, Z. Q. Qi, R. A. Richter, E. A. Strychalski, L. Sun, Y. Suzuki, B. Tsvetanova, K. S. Wise, H. O. Smith, J. I. Glass, C. Merryman, D. G. Gibson, J. C. Venter, Science 2016, 351, aad6253.

[2] a) M. M. Hanczyc, S. M. Fujikawa, J. W. Szostak, Science 2003, 302, 618-622; b) T. F. Zhu, J. W. Szostak, J. Am. Chem. Soc. 2009, 131, 5705-5713.
[3] a) T. Oberholzer, R. Wick, P. L. Luisi, C. K. Biebricher, Biochem. Biophys. Res. Commun. 1995, 207, 250-257; b) K. Adamala, J. W. Szostak, Science 2013, 342, 1098-1100.

[4] I. A. Chen, K. Salehi-Ashtiani, J. W. Szostak, J. Am. Chem. Soc. 2005, 127, 13213-13219.

[5] a) G. Steinberg-Yfrach, J. L. Rigaud, E. N. Durantini, A. L. Moore, D. Gust, T. A. Moore, Nature 1998, 392, 479-482; b) H.J. Choi, C. D. Montemagno, Nano Lett. 2005, 5, 2538-2542; c) X. Feng, Y. Jia, P. Cai, J. Fei, J. Li, ACS Nano 2016, 10, 556-561; d) G. Nordlund, P. Brzezinski, C. v. Ballmoos, Nat. Commun. 2014, 5,4303 .

[6] M. Weiss, J. P. Frohnmayer, L. T. Benk, B. Haller, J.-W. Janiesch, T. Heitkamp, M. Börsch, R. B. Lira, R. Dimova, R. Lipowsky, E. Bodenschatz, J.-C. Baret, T. Vidakovic-Koch, K. Sundmacher, I. Platzman, J. P. Spatz, Nat. Mater. 2018, 17, 89-96.

[7] L. Otrin, N. Marušič, C. Bednarz, T. Vidaković-Koch, I. Lieberwirth, K. Landfester, K. Sundmacher, Nano Lett. 2017, 17, $6816-6821$.

[8] a) P. Walde, S. Ichikawa, Biomol. Eng. 2001, 18, 143-177; b) J. Lim, O. Caen, J. Vrignon, M. Konrad, V. Taly, J.-C. Baret, Biomicrofluidics 2015, 9, 034101; c) M. Nijemeisland, L. K. E. A. Abdelmohsen, W. T. S. Huck, D. A. Wilson, J. C. M. van Hest, ACS Cent. Sci. 2016, 2, 843-849; d) R. J. R. W. Peters, M. Marguet, S. Marais, M. W. Fraaije, J. C. M. van Hest, S. Lecommandoux, Angew. Chem. Int. Ed. 2014, 53, 146-150; Angew. Chem. 2014, 126, 150-154; e) Y. Elani, R. V. Law, O. Ces, Nat. Commun. 2014, 5, 5305.

[9] a) P. H. Opgenorth, T. P. Korman, J. U. Bowie, Nat. Chem. Biol. 2016, 12, 393-395; b) P. H. Opgenorth, T. P. Korman, L. Iancu, J. U. Bowie, Nat. Chem. Biol. 2017, 13, 938; c) T. P. Korman, P. H. Opgenorth, J. U. Bowie, Nat. Commun. 2017, 8, 15526.

[10] T. Schwander, L. Schada von Borzyskowski, S. Burgener, N. S. Cortina, T. J. Erb, Science 2016, 354, 900 - 904.

[11] T. J. Erb, P. R. Jones, A. Bar-Even, Curr. Opin. Chem. Biol. 2017, $37,56-62$.

[12] J. Sun, J. G. Jeffryes, C. S. Henry, S. D. Bruner, A. D. Hanson, Metab. Eng. 2017, 44, 150-159.

[13] a) I. A. Chen, J. W. Szostak, Biophys. J. 2004, 87, 988-998; b) P. Stano, P. L. Luisi, Chem. Commun. 2010, 46, 3639-3653.

[14] K. Kurihara, M. Tamura, K. Shohda, T. Toyota, K. Suzuki, T. Sugawara, Nat. Chem. 2011, 3, 775-781.

[15] a) C. K. Haluska, K. A. Riske, V. Marchi-Artzner, J. M. Lehn, R. Lipowsky, R. Dimova, Proc. Natl. Acad. Sci. USA 2006, 103, 15841-15846; b) H. Terasawa, K. Nishimura, H. Suzuki, T. Matsuura, T. Yomo, Proc. Natl. Acad. Sci. USA 2012, 109, $5942-$ 5947; c) M. J. Kyoung, Y. X. Zhang, J. J. Diao, S. Chu, A. T. Brunger, Nat. Protoc. 2013, 8, 1-16; d) B. R. Lentz, Eur. Biophys. J. 2007, 36, 315-326; e) Y. Suzuki, K. H. Nagai, A. Zinchenko, T. Hamada, Langmuir 2017, 33, 2671-2676.

[16] M. C. F. A. C. Jewett, Curr. Opin. Biotechnol. 2010, 21, 697-703.

[17] N. Ichihashi, K. Usui, Y. Kazuta, T. Sunami, T. Matsuura, T. Yomo, Nat. Commun. 2013, 4, 2494.

[18] a) C. P. Brangwynne, C. R. Eckmann, D. S. Courson, A. Rybarska, C. Hoege, J. Gharakhani, F. Julicher, A. A. Hyman, Science 2009, 324, 1729-1732; b) C. P. Brangwynne, T. J. Mitchison, A. A. Hyman, Proc. Natl. Acad. Sci. USA 2011, 108, 4334-4339; c) P. Li, S. Banjade, H. C. Cheng, S. Kim, B. Chen, L. Guo, M. Llaguno, J. V. Hollingsworth, D. S. King, S. F. Banani, P. S. Russo, Q. X. Jiang, B. T. Nixon, M. K. Rosen, Nature 2012, 483, 336-340; d) X. L. Su, J. A. Ditlev, E. F. Hui, W. M. Xing, S. Banjade, J. Okrut, D. S. King, J. Taunton, M. K. Rosen, R. D. Vale, Science 2016, 352, 595-599.

[19] a) S. Saha, C. A. Weber, M. Nousch, O. Adame-Arana, C. Hoege, M. Y. Hein, E. Osborne-Nishimura, J. Mahamid, M. Jahnel, L. Jawerth, A. Pozniakovski, C. R. Eckmann, F. Julicher, A. A. Hyman, Cell 2016, 166, 1572-1584; b) A. Patel, H. O. Lee, L. Jawerth, S. Maharana, M. Jahnel, M. Y. Hein, S. Stoynov, J. 
Mahamid, S. Saha, T. M. Franzmann, A. Pozniakovski, I. Poser, N. Maghelli, L. A. Royer, M. Weigert, E. W. Myers, S. Grill, D. Drechsel, A. A. Hyman, S. Alberti, Cell 2015, 162, 1066-1077.

[20] D. Zwicker, R. Seyboldt, C. A. Weber, A. A. Hyman, F. Jülicher, Nat. Phys. 2017, 13, 408-413.

[21] T. Wollert, C. Wunder, J. Lippincott-Schwartz, J. H. Hurley, Nature 2009, 458, $172-177$.

[22] M. Osawa, H. P. Erickson, Proc. Natl. Acad. Sci. USA 2013, 110, $11000-11004$

[23] T. Härtel, P. Schwille, Front. Microbiol. 2014, 5, 257.

[24] S. Jun, A. Wright, Nat. Rev. Microbiol. 2010, 8, 600-607.

[25] E. C. Garner, C. S. Campbell, D. B. Weibel, R. D. Mullins, Science 2007, 315, 1270-1274.

[26] K. Zieske, G. Chwastek, P. Schwille, Angew. Chem. Int. Ed. 2016, 55, 13455-13459; Angew. Chem. 2016, 128, 13653-13657.

[27] M. Schmick, P. I. H. Bastiaens, Cell 2014, 156, 1132-1138.

[28] M. Schmick, A. Kraemer, P. I. H. Bastiaens, Trends Cell Biol. 2015, 25, 190-197.

[29] a) P. Bastiaens, M. Caudron, P. Niethammer, E. Karsenti, Trends Cell Biol. 2006, 16, 125-134; b) C. I. Maeder, M. A. Hink, A Kinkhabwala, R. Mayr, P. I. H. Bastiaens, M. Knop, Nat. Cell Biol. 2007, 9, 1319-1326.

[30] A. Koseska, P. I. Bastiaens, EMBO J. 2017, 36, 568-582.

[31] A. B. Theberge, F. Courtois, Y. Schaerli, M. Fischlechner, C. Abell, F. Hollfelder, W. T. Huck, Angew. Chem. Int. Ed. 2010, 49 , 5846-5868; Angew. Chem. 2010, 122, 5982-6005.

[32] a) D. C. Duffy, J. C. McDonald, O. J. A. Schueller, G. M. Whitesides, Anal. Chem. 1998, 70, 4974-4984; b) Y. Xia, G. M. Whitesides, Annu. Rev. Mater. Sci. 1998, 28, 153-184.

[33] L. Mazutis, J. C. Baret, A. D. Griffiths, Lab Chip 2009, 9, 2665 2672.

[34] A. R. Abate, T. Hung, P. Mary, J. J. Agresti, D. A. Weitz, Proc. Natl. Acad. Sci. USA 2010, 107, 19163-19166.

[35] a) Y. Kazayama, T. Teshima, T. Osaki, S. Takeuchi, T. Toyota, Anal. Chem. 2016, 88, 1111-1116; b) T. Robinson, P. E. Verboket, K. Eyer, P. S. Dittrich, Lab Chip 2014, 14, 2852-2859.

[36] B. Kubsch, T. Robinson, R. Lipowsky, R. Dimova, Biophys. J. 2016, 110, 2581-2584.

[37] J. Shin, V. Noireaux, ACS Synth. Biol. 2012, 1, 29-41.

[38] R. Sachse, D. Wüstenhagen, M. Šamalíková, M. Gerrits, F. F. Bier, S. Kubick, Eng. Life Sci. 2013, 13, 39-48.

[39] J. Shin, P. Jardine, V. Noireaux, ACS Synth. Biol. 2012, 1, $408-$ 413.

[40] M. Stech, H. Merk, J. A. Schenk, W. F. M. Stöcklein, D. A. Wüstenhagen, B. Micheel, C. Duschl, F. F. Bier, S. Kubick, J. Biotechnol. 2013, 164, 220-231.
[41] a) S.-i. M. Nomura, K. Tsumoto, T. Hamada, K. Akiyoshi, Y. Nakatani, K. Yoshikawa, ChemBioChem 2003, 4, 1172-1175; b) V. Noireaux, A. Libchaber, Proc. Natl. Acad. Sci. USA 2004, 101, 17669-17674.

[42] a) F. Courtois, L. F. Olguin, G. Whyte, D. Bratton, W. T. S. Huck, C. Abell, F. Hollfelder, ChemBioChem 2008, 9, 439-446; b) P. S. Dittrich, M. Jahnz, P. Schwille, ChemBioChem 2005, 6, 811-814.

[43] M. Nallani, M. Andreasson-Ochsner, C.-W. D. Tan, E.-K. Sinner, Y. Wisantoso, S. Geifman-Shochat, W. Hunziker, Biointerphases 2011, 6, 153-157.

[44] T. Y. D. Tang, D. v. Swaay, A. deMello, J. L. R. Anderson, S. Mann, Chem. Commun. 2015, 51, 11429-11432.

[45] a) E. Sokolova, E. Spruijt, M. M. K. Hansen, E. Dubuc, J. Groen, V. Chokkalingam, A. Piruska, H. A. Heus, W. T. S. Huck, Proc. Natl. Acad. Sci. USA 2013, 110,11692-11697; b) P. Torre, C. D. Keating, S. S. Mansy, Langmuir 2014, 30, 5695-5699.

[46] M. Stech, A. K. Brödel, R. B. Quast, R. Sachse, S. Kubick, Adv. Biochem. Eng./Biotechnol. 2013, 137, 67-102.

[47] C. Martino, A. J. deMello, Interface Focus 2016, 6, 20160011.

[48] H. Jia, M. Heymann, F. Bernhard, P. Schwille, L. Kai, Nat. Biotechnol. 2017, 39, 199-205.

[49] M. C. Jewett, B. R. Fritz, L. E. Timmerman, G. M. Church, Mol. Syst. Biol. 2013, 9, 678.

[50] F. Caschera, V. Noireaux, Curr. Opin. Chem. Biol. 2014, 22, $85-$ 91

[51] I. Otero-Muras, J. R. Banga, ACS Synth. Biol. 2017, 6, 11801193.

[52] S. Rollié, M. Mangold, K. Sundmacher, Chem. Eng. Sci. 2012, 69, $1-29$.

[53] E. Schneider, M. Mangold, Biosystems 2018, 165, 8-21.

[54] A. M. Tayar, S. S. Daube, R. H. Bar-Ziv, Curr. Opin. Chem. Biol. 2017, 40,37-46.

[55] a) New Directions. The Ethics of Synthetic Biology and Emerging Technologies. Executive Summary and Recommendations, Vol. 16, United States, Presidential Commission for the Study of Bioethical Issues, Washington, D. C. 2010; b) Emerging Policy Issues in Synthetic Biology, OECD, Paris, 2014.

[56] Biosecurity - Freedom and Responsibility of Research, Deutscher Ethikrat, 2014

[57] B. Baertschi, Environ. Values 2012, 21, 5-18.

Manuskript erhalten: 21. Februar 2018

Veränderte Fassung erhalten: 3. April 2018

Akzeptierte Fassung online: 11. Mai 2018

Endgültige Fassung online: 17. September 2018 